\title{
Comparison of the effects on pupil size and accommodation of three regimens of topical dapiprazole
}

Charles S Wilcox, Jon F Heiser, Anna Mae Crowder, Nancy J Wassom, Barbara B Katz, Jacqueline L Dale
Pharmacology

Research Institute, Irvine, California, USA

C S Wilcox

J F Heiser

A M Crowder

N J Wassom

B B Katz

J L Dale

Correspondence to: Charres S Wilcox, $\mathrm{PhD}$ 19712 MacArthur Boulevard, Suite 220, Irvine, Boulevard, Suite 220, Ir

Accepted for publication 26 January 1995

\begin{abstract}
Background-Patients who have their pupils dilated for an eye examination traditionally have to wait several hours before their pupils return to normal size and their blurred vision (caused by paralysis of accommodation) resolves. Earlier studies with dapiprazole have demonstrated an accelerated reversal of dilatation.

Methods-Three regimens of dapiprazole were studied to determine the effects on pupil diameter and accommodation after mydriasis produced by $2.5 \%$ phenylephrine and $0.5 \%$ tropicamide. Test regimens included one drop and $1+1$ drop regimens, compared with a $2+2$ drop reference regimen. Dapiprazole was administered in one eye and placebo in the other. Mean change from baseline was analysed for pupil diameter and accommodation at various time points after drug administration. Also, for the same variables, $90 \%$ confidence intervals for the areas under the curve (AUC) were computed.
\end{abstract}

Results-Both test regimens were equivalent to the reference regimen on the basis of mean change from baseline for pupil diameter and accommodation at individual time points, and for the mean AUC. Most signs and symptoms (injection, stinging, burning, lid oedema, and ptosis) were less frequent in the test regimen treated eyes. There was no significant interaction between regimen and eye colour.

Conclusion-This study indicates that a lower dosage (for example, one drop) is also efficacious and has the added benefit of fewer side effects.

(Br f Ophthalmol 1995; 79: 544-548)

Dapiprazole $\quad 0.5 \% \quad$ (Rev-Eyes; Angelini Pharmaceuticals Inc. Rev-Eyes is manufactured by Abbott Laboratories and marketed by Storz Ophthalmics Inc) was introduced in the United States in 1991 in response to the need for a convenient method of reversing iatrogenically induced mydriasis. Dapiprazole is an $\alpha$ adrenergic blocking agent administered topically. Its chemical formula is $5,6,7,8-$ tetrahydro-3-[2-(4-o-tolyl-1-piperazinyl) ethyl]s-triazolo [4,3-a] pyridine hydrochloride. While its primary action is the reversal of mydriasis induced by sympathomimetic agents, such as phenylephrine, and parasympatholytics, such as tropicamide, dapiprazole also shows a beneficial effect on loss of accommodation, ${ }^{1}$ although the exact mechanism for its effect on accommodation is unknown. In most patients, reversal of mydriasis and improvement in accommodation begin within 15 minutes of administration of the drug.

Since its introduction, dapiprazole has been used in a $2+2$ regimen, two drops followed by two more drops 5 minutes later. In clinical practice, returning to dose the patient for a second time in 5 minutes can sometimes be troublesome for both the ophthalmologist and the patient. After a review of the literature on dapiprazole, Doughty and Lyle ${ }^{2}$ suggested that further studies should be conducted to investigate the optimum dosing regimen. In addition, the capacity of the cul de sac has been estimated as $0.025 \mathrm{ml},{ }^{3}$ suggesting that significant washout of a two drop dose may occur; furthermore, known side effects of dapiprazole may also be reduced by a regimen with fewer drops.

This study explored the comparative efficacy of three regimens of dapiprazole. Regimens were compared with respect to reversal of mydriasis, improvement in accommodation, and incidence of side effects. Known eye colour effects (slower reversal of mydriasis in patients with dark irides) were controlled for in the experimental design.

\section{STUDY OBJECTIVE}

The objective of this study was to demonstrate the equivalence of two test regimens to a 'reference' regimen of $0.5 \%$ dapiprazole in their ability to reverse mydriasis induced by $2 \cdot 5 \%$ phenylephrine (Neosynephrine, Winthrop) and $0.5 \%$ tropicamide (Bausch and Lomb). A secondary objective was to evaluate and compare the subject's ability to tolerate the three regimens. No previous study had compared the effects of different regimens of $0.5 \%$ dapiprazole.

\section{Materials and methods}

A clinically significant mean change from baseline for pupil diameter, between reference and test compounds, was $0.5 \mathrm{~mm}$. Data from a previous study indicated a within (intra) subject variance of $0 \cdot 81$. The computed sample size was 55 subjects. In order to allocate an 
equal number of subjects to three regimens, a sample size of 60 was chosen.

This was a double masked, placebo controlled, crossover study. Subjects participated in three study sessions, each at least 5 days apart. Treatment sequence group and the eye to be treated were randomised and balanced within eye colour (brown or other). After baseline examinations, each subject had both eyes dilated with $2.5 \%$ phenylephrine and $0.5 \%$ tropicamide. After 1 hour, one eye was treated with one of three dapiprazole regimens and the other eye was treated with placebo (the inactive ingredients of the dapiprazole formulation). The three regimens are: one drop (' 1 ' drop test regimen); one drop plus one drop 5 minutes later (' $1+1$ ' drop test regimen); two drops plus two drops 5 minutes later (' $2+2$ ' drops reference regimen).

To ensure masking of the investigator, preparation and administration of study regimens were performed by an individual who was not involved in subsequent evaluations.

Baseline examinations included general medical and ocular histories. An external ophthalmic examination and a slit-lamp examination of the anterior segment of the eye were also conducted, along with pupil diameter, distance visual acuity, and near point of accommodation. Throughout the study the measurements of pupil diameter, distance visual acuity, and near point of accommodation were performed before (hour 0 ) and after instillation of the study drug at time points ranging from 15 minutes after administration to 6 hours.

In all measurements, the right eye was evaluated first and then the left eye. Pupil diameter was measured by having subjects focus on a visual chart at a distance of 20 feet, and horizontal pupil measurement was taken to the nearest $0.5 \mathrm{~mm}$ using a pupillometric scale; all measurements were performed by the same licensed clinician, using a standard (Bausch and Lomb) pupil diameter gauge. The examination rooms used standardised ambient illumination.

Near point of accommodation was measured with the subject corrected for distance. The Prince rule was attached to the phoropter. The Prince rule is a device for determining the near point and amplitude of accommodation, consisting of a steel tape scaled in dioptres on one side and in millimetres on the other. One end of the tape is held against the lower orbital margin, and a test chart is moved along the tape, towards the eye, until a blur is noticed. ${ }^{4}$ The $20 / 30$ reduced Snellen line on the Rosenbaum pocket vision screener card (for example, a reduced Snellen chart for near vision) was used. The card was positioned at the closest distance to the eye and then moved away until the subject could read the 20/30 line. This step was performed three times at all measurements (however, in one instance it was inadvertently done only once at baseline). Each time the distance was noted in centimetres and converted to dioptres. If the subject could not read the 20/30 line at the

farthest distance (50 centimetres) on the
Prince rule, a line that was visible at that distance was identified and recorded (for example, $20 / 40$ at $50 \mathrm{~cm}$ ).

Corrected visual acuity was measured using the standard Snellen chart at 20 feet. Safety was assessed by recording signs and symptoms of the external eye and periocular tissues. The severity of adverse events and their relation to the study drug were routinely recorded in a standardised manner.

The baseline measurements used for comparison of pupil diameter and accommodation between the test regimens and the reference regimen were the pre-mydriasis measurements. Pupil diameter and accommodation were measured at baseline, 0,15 , and $30 \mathrm{~min}$ utes and at 1,2, 4, and 6 hours after treatment with dapiprazole or placebo.

The primary efficacy variable was mean pupil diameter change from baseline. A clinically significant difference between regimens at individual time points was $\geqslant 0.5 \mathrm{~mm}$. Mean change from baseline was expressed as a positive number, since the pupil size increased after the mydriatic was administered. A secondary efficacy variable was mean accommodation loss from baseline. A clinically significant difference between regimens at individual time points was defined as $\geqslant 1$ dioptre. Mean change from baseline was expressed as a negative number, since accommodation decreased after the cycloplegic was administered.

Area under the curve (AUC) for mean pupil diameter was computed from 0 hours (premydriasis) to 6 hours (post treatment). The AUC was computed in $\mathrm{mm} \times \mathrm{h}$. A clinically significant difference between regimens for AUC was $3 \mathrm{~mm} \times \mathrm{h}(0.5 \mathrm{~mm} \times 6$ hours $)$. Likewise, AUC for mean accommodation was computed from 0 to 6 hours. The AUC was computer in $\mathrm{D} \times \mathrm{h}$. A clinically significant difference between regimens for AUC was $6 \mathrm{D} \times \mathrm{h}(1 \mathrm{D}$ $\times 6$ hours). ${ }^{\star}$

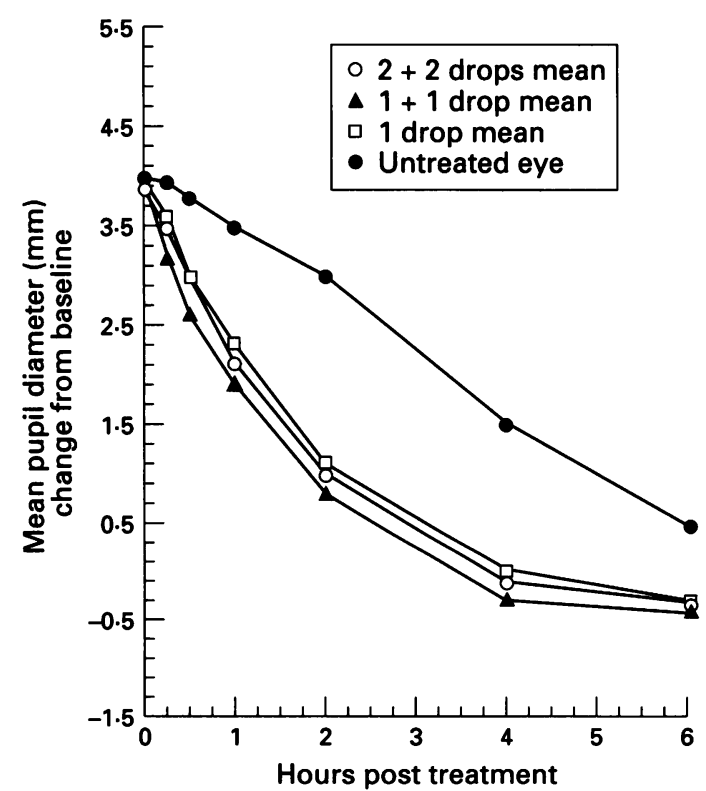

Figure 1 Plot of means of pupil diameter for treated and untreated eyes. Comparison between each of three treatment regimens and untreated eyes. reported for treated eyes for each of the three dapiprazole regimen groups. The smaller is the absolute value of the was the recovery from loss of accommodation over the 6 hour period. 
Table 1 Pupil diameter ( $\mathrm{mm}$ ): mean change from baseline

\begin{tabular}{|c|c|c|c|c|c|c|c|c|c|c|c|c|}
\hline & \multirow{2}{*}{\multicolumn{2}{|c|}{$\begin{array}{l}\text { Reference } \\
2+2 \text { drops } \\
(2+2)\end{array}$}} & \multirow{2}{*}{\multicolumn{2}{|c|}{$\begin{array}{l}\text { Test } \\
1 \text { drop } \\
\text { (1) }\end{array}$}} & \multicolumn{3}{|c|}{$\begin{array}{l}\text { Estimate test-reference } \\
(1-2+2)\end{array}$} & \multirow{2}{*}{\multicolumn{2}{|c|}{$\begin{array}{l}\text { Test } 1+1 \\
\text { drop } \\
(1+1)\end{array}$}} & \multicolumn{3}{|c|}{$\begin{array}{l}\text { Estimate test-reference } \\
(1+1-2+2)\end{array}$} \\
\hline & & & & & \multirow[b]{2}{*}{ Mean } & \multirow[b]{2}{*}{$S E$} & \multirow{2}{*}{$\begin{array}{l}90 \% \\
\text { Confidence } \\
\text { limits }\end{array}$} & & & \multirow[b]{2}{*}{ Mean } & \multirow[b]{2}{*}{$S E$} & \multirow{2}{*}{$\begin{array}{l}90 \% \\
\text { Confidence } \\
\text { limits }\end{array}$} \\
\hline & Mean & $S E$ & Mean & $S E$ & & & & Mean & $S E$ & & & \\
\hline $\begin{array}{l}15 \text { Minutes } \\
30 \text { Minutes } \\
1 \text { Hour } \\
2 \text { Hours } \\
4 \text { Hours } \\
6 \text { Hours } \\
\text { AUC }\end{array}$ & $\begin{array}{r}3 \cdot 5 \\
3 \cdot 0 \\
2 \cdot 1 \\
1 \cdot 0 \\
-0 \cdot 1 \\
-0.3 \\
6 \cdot 3\end{array}$ & $\begin{array}{l}0 \cdot 1 \\
0 \cdot 1 \\
0 \cdot 1 \\
0 \cdot 1 \\
0 \cdot 1 \\
0 \cdot 1 \\
0 \cdot 4\end{array}$ & $\begin{array}{r}3 \cdot 6 \\
3 \cdot 0 \\
2 \cdot 3 \\
1 \cdot 1 \\
0 \cdot 0 \\
-0.3 \\
6 \cdot 8\end{array}$ & $\begin{array}{l}0 \cdot 1 \\
0.1 \\
0 \cdot 1 \\
0 \cdot 2 \\
0.1 \\
0 \cdot 1 \\
0.5\end{array}$ & $\begin{array}{l}0.2 \\
0.0 \\
0 \cdot 2 \\
0 \cdot 1 \\
0 \cdot 1 \\
0.0 \\
0.5\end{array}$ & $\begin{array}{l}0 \cdot 1 \\
0 \cdot 1 \\
0 \cdot 2 \\
0 \cdot 1 \\
0 \cdot 1 \\
0 \cdot 1 \\
0 \cdot 5\end{array}$ & $\begin{array}{c}-0.07,0.38 \\
-0.19,0.21 \\
-0.10,0.40 \\
-0.17,0.29 \\
-0.10,0.28 \\
-0.14,0.20 \\
0.27,1.2\end{array}$ & $\begin{array}{r}3 \cdot 2 \\
2.6 \\
1.9 \\
0 \cdot 8 \\
-0.3 \\
-0.4 \\
5.6\end{array}$ & $\begin{array}{l}0 \cdot 1 \\
0 \cdot 1 \\
0 \cdot 1 \\
0 \cdot 1 \\
0 \cdot 1 \\
0 \cdot 1 \\
0.4\end{array}$ & $\begin{array}{l}-0.2 \\
-0.4 \\
-0.2 \\
-0.3 \\
-0.2 \\
-0.1 \\
-0.7\end{array}$ & $\begin{array}{l}0 \cdot 1 \\
0 \cdot 1 \\
0 \cdot 1 \\
0 \cdot 1 \\
0 \cdot 1 \\
0 \cdot 1 \\
0 \cdot 4\end{array}$ & $\begin{array}{l}-0.47,-0.02 \\
-0.56,-0.16 \\
-0.45,0.06 \\
-0.48,-0.02 \\
-0.40,-0.02 \\
-0.24,0.10 \\
-1.5,0.1\end{array}$ \\
\hline
\end{tabular}

Equivalence was established by the confidence limit approach of Westlake. ${ }^{5}$ Two one sided $t$ tests for equivalence between regimens have been shown to be the same as $90 \%$ confidence limits on their difference. The study was analysed as a crossover study with regimen and period as within subject factors and eye colour as a between subject factor. The interactions of eye colour with regimen and period were also included. Estimates of the differences between regimens and their standard errors were computed from the analysis and used to calculate the $90 \%$ confidence limits. Only comparisons among dapiprazole treated eyes are included in the analyses for equivalence in regimens. Comparisons of treated and untreated eyes were used to test for efficacy which has been previously established. ${ }^{6-8}$

\section{Results of comparability testing}

\section{SUBJECTS}

Sixty two subjects were enrolled. Normal volunteers, including men and women between 18-40 years of age were recruited at one investigational site in Southern California (Pharmacology Research Institute, 19712 MacArthur Boulevard, Suite 220, Irvine, CA 92715-2407, USA). The principal investigator was Jon $\mathrm{F}$ Heiser, MD.

Sixty two subjects, who were evaluated against the enrolment criteria and signed an informed consent form participated in the study. In order to be included in the efficacy analysis, subjects had to be present for all three treatment periods. There were two subjects who did not complete all three periods. Those subjects were replaced. Data from the replaced subjects were excluded from the efficacy analysis but included in the safety analysis.

The 60 evaluable subjects ranged in age from 18 to 37 (mean 25.4) years. Forty six men $(77 \%)$ and 14 women (23\%) participated in the study over a period of approximately 6 weeks in the summer of 1992 .

BASELINE EQUIVALENCE

There were no significant differences at baseline in age, sex, accommodation, or visual acuity for eye colour or sequence group. There was a significant difference $(p=0.024)$ at baseline in mean pupil diameter for eye colours, with smaller pupils found in subjects with brown eyes. All non-white subjects had brown eyes; therefore, there was a significant eye colour effect, but there was no evidence of differences in racial composition of the sequence groups.

\section{PUPIL DIAMETER}

For individual time points, comparing regimens on the basis of mean pupil diameter change from baseline, the clinically significant difference of $0.5 \mathrm{~mm}$ was not included in the $90 \%$ confidence intervals; therefore, the one drop regimen was equivalent to the $2+2$ drop regimen.

The $1+1$ regimen was equivalent to the $2+2$ regimen in reversing mydriasis at all time points other than 30 minutes, where the $90 \%$ confidence interval did include the clinically significant difference. At the 30 minute point, the reversal with the $1+1$ regimen exceeded that of the $2+2$ regimen. Figure 1 represents the mean changes from baseline for pupil diameter data, illustrating mean comparisons between each of the three treatment regimens as well as the untreated eyes.

The AUC is a composite of the data from each of the individual time points. In analysing the mean pupil diameter AUC, the areas were $6.8 \mathrm{~mm} \times \mathrm{h}$ for the 1 drop regimen, $5.6 \mathrm{~mm} \times \mathrm{h}$ for the $1+1$ drop regimen, and $6.3 \mathrm{~mm} \times \mathrm{h}$ for the $2+2$ regimen. Computing $90 \%$ confidence intervals for the difference in the mean AUC between the test and the reference regimens, both test regimens were found to be equivalent to the reference regimen. Table 1 summarises

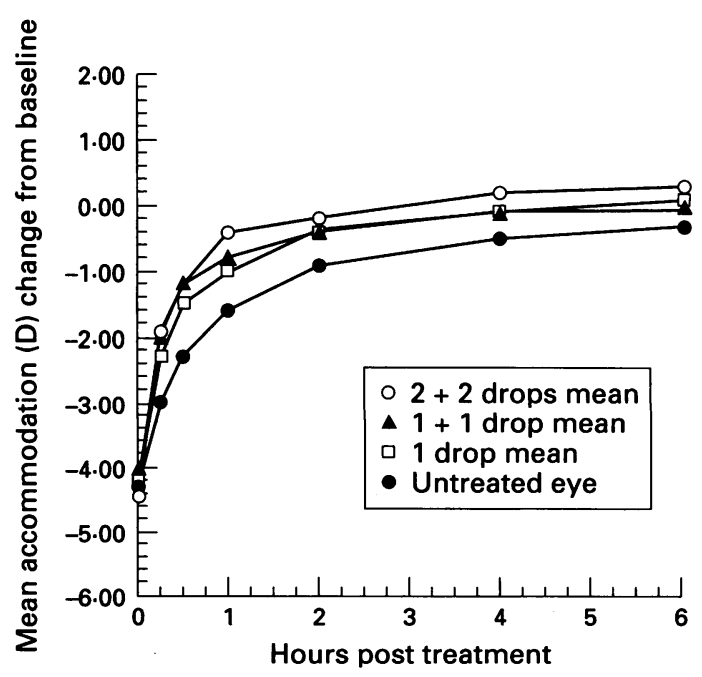

Figure 2 Plot of mean changes from baseline for accommodation data. Comparison between each of the three treatment regimens and untreated eyes. 
Table 2 Accommodation (D): mean change from baseline

\begin{tabular}{|c|c|c|c|c|c|c|c|c|c|c|c|c|}
\hline & \multirow{2}{*}{\multicolumn{2}{|c|}{$\begin{array}{l}\text { Reference } \\
2+2 \text { drops } \\
(2+2)\end{array}$}} & \multirow{2}{*}{\multicolumn{2}{|c|}{$\begin{array}{l}\text { Test } \\
1 \text { drop } \\
\text { (1) }\end{array}$}} & \multicolumn{3}{|c|}{$\begin{array}{l}\text { Estimate test-reference } \\
(1-2+2)\end{array}$} & \multirow{2}{*}{\multicolumn{2}{|c|}{$\begin{array}{l}\text { Test } 1+1 \\
\text { drop } \\
(1+1)\end{array}$}} & \multicolumn{3}{|c|}{$\begin{array}{l}\text { Estimate test-reference } \\
(1+1-2+2)\end{array}$} \\
\hline & & & & & \multirow[b]{2}{*}{ Mean } & \multirow[b]{2}{*}{$S E$} & \multirow{2}{*}{$\begin{array}{l}90 \% \\
\text { Confidence } \\
\text { limits }\end{array}$} & & & \multirow[b]{2}{*}{ Mean } & \multirow[b]{2}{*}{$S E$} & \multirow{2}{*}{$\begin{array}{l}90 \% \\
\text { Confidence } \\
\text { limits }\end{array}$} \\
\hline & Mean & $S E$ & Mean & $S E$ & & & & Mean & $S E$ & & & \\
\hline $\begin{array}{l}15 \text { Minutes } \\
30 \text { Minutes } \\
1 \text { Hour } \\
2 \text { Hours } \\
4 \text { Hours } \\
6 \text { Hours } \\
\text { AUC }\end{array}$ & $\begin{array}{r}-1.9 \\
-1.2 \\
-0.4 \\
-0.2 \\
0.2 \\
0.3 \\
-2.8\end{array}$ & $\begin{array}{l}0 \cdot 2 \\
0 \cdot 2 \\
0 \cdot 1 \\
0 \cdot 1 \\
0 \cdot 1 \\
0 \cdot 1 \\
0 \cdot 3\end{array}$ & $\begin{array}{r}-2.3 \\
-1.5 \\
-1.0 \\
-0.4 \\
-0 \cdot 1 \\
0.1 \\
-4.0\end{array}$ & $\begin{array}{l}0 \cdot 2 \\
0 \cdot 2 \\
0 \cdot 2 \\
0 \cdot 1 \\
0 \cdot 1 \\
0 \cdot 1 \\
0 \cdot 5\end{array}$ & $\begin{array}{l}-0.4 \\
-0.3 \\
-0.6 \\
-0.2 \\
-0.3 \\
-0.2 \\
-1.3\end{array}$ & $\begin{array}{l}0 \cdot 2 \\
0 \cdot 2 \\
0 \cdot 2 \\
0 \cdot 2 \\
0 \cdot 1 \\
0 \cdot 1 \\
0.5\end{array}$ & $\begin{array}{l}-0.71,-0.06 \\
-0.63,-0.04 \\
-0.86,-0.30 \\
-0.45,-0.01 \\
-0.48,-0.07 \\
-0.43,-0.04 \\
-2.0,-0.53\end{array}$ & $\begin{array}{r}-2 \cdot 0 \\
-1 \cdot 2 \\
-0.8 \\
-0.4 \\
-0 \cdot 1 \\
0 \cdot 0 \\
-3 \cdot 4\end{array}$ & $\begin{array}{l}0 \cdot 2 \\
0 \cdot 1 \\
0 \cdot 1 \\
0 \cdot 1 \\
0 \cdot 1 \\
0 \cdot 1 \\
0.4\end{array}$ & $\begin{array}{l}-0.1 \\
-0.1 \\
-0.3 \\
-0.2 \\
-0.3 \\
-0.3 \\
-0.7\end{array}$ & $\begin{array}{l}0 \cdot 2 \\
0 \cdot 2 \\
0 \cdot 1 \\
0 \cdot 1 \\
0 \cdot 1 \\
0 \cdot 1 \\
0 \cdot 4\end{array}$ & $\begin{array}{l}-0.42,0.24 \\
-0.36,0.23 \\
-0.61,-0.04 \\
-0.41,0.03 \\
-0.49,-0.08 \\
-0.50,-0.11 \\
-1 \cdot 4,0.1\end{array}$ \\
\hline
\end{tabular}

the mean change from baseline for pupil diameter data and the AUC.

\section{ACCOMMODATION}

Comparing regimens on the basis of mean accommodation change from baseline for each individual time point, the clinically significant difference of 1.0 dioptre was not included in the $90 \%$ confidence intervals; therefore, the one drop and the $1+1$ drop regimens were equivalent to the $2+2$ drop regimen. Figure 2 represents the mean changes from baseline for accommodation data, illustrating mean comparisons between each of the three treatment regimens as well as the untreated eyes.

The AUCs for accommodation were $-4 \cdot 0$ $\mathrm{D} \times \mathrm{h}$ for the one drop regimen, $-3.4 \mathrm{D} \times \mathrm{h}$ for the $1+1$ drop regimen, and $-2 \cdot 8 \mathrm{D} \times \mathrm{h}$ for the $2+2$ regimen (greater accommodation, but not significant (eye $\times$ eye colour)). Computing $90 \%$ confidence intervals for the difference in mean AUC between the test and reference regimen, both test regimens were found to be equivalent to the reference regimen. Table 2 summarises the mean change from baseline for accommodation data and the AUC.

\section{EYE COLOUR}

Eye colour was a significant effect in the analysis for both pupil diameter and accommodation. The eye colour effect was generally seen by 1 hour and persisted until 6 hours; this effect was seen in the AUC as well. Differences in effect due to eye colour were consistent across regimens (that is, there were no significant interactions).

\section{SIGNS AND SYMPTOMS}

In most instances, signs and symptoms were less frequent in eyes treated with the test regimens than in eyes treated with the reference regimen. Most signs and symptoms were mild, except conjunctival injection, where some reports were of moderate severity. Events usually subsided on the study day without treatment. The most common and expected side effect was conjunctival injection, occurring in $98 \%$ of treated eyes in each regimen. Injection is expected as it is a pharmacological action of dapiprazole, an $\alpha$ antagonist. Table 3 summarises the primary signs and symptoms noted during the course of the study.

Clinically significant decreases in visual acuity, defined as more than a one line decrease on the Snellen chart, occurred in fewer of the eyes treated with dapiprazole $(14.7 \%$ or 27 eyes) than in eyes treated with placebo $(23.5 \%$ or 43 eyes).

\section{Discussion}

For patient comfort and convenience, Nyman and Keates ${ }^{9}$ have suggested that a safe, effective miotic to reverse diagnostic mydriasis would be welcomed by health care professionals and patients alike. The safety and efficacy of dapiprazole has been demonstrated in previous studies of the $2+2$ regimen alone.

This study supports the safety and efficacy of two regimens with reduced dosages. The one drop and $1+1$ drop regimens were found to be equivalent to the currently marketed $2+2$ drop regimen at individual time points, comparing mean pupil diameter and accommodation change from baseline. The AUC analysis also supports the equivalence of the test and reference regimens. On the basis of reversal of mydriasis, the two test regimens of dapiprazole are efficacious alternatives to the $2+2$ regimen. The perceived patient benefit from the more rapid reversal of mydriasis is obviously subjective and, of course, variable.

The duration of pupil dilatation with the combination of phenylephrine and tropicamide has been reported to be 7 hours, and the duration of significantly reduced accommodative amplitude to be 3 hours. ${ }^{9}$ During this time, some patients who are sensitive may experience the discomfort of photophobia and glare. Allinson ${ }^{8}$ has reported that 'reversing diagnostic mydriasis as rapidly as possible increases patient comfort'. Johnson et al concluded that the perceived benefit to the patient comes from the increased rate of recovery of accommodation, rather than from reversal of mydriasis. ${ }^{7}$

Table 3 Signs and symptoms after dapiprazole

\begin{tabular}{llll}
\hline & \multicolumn{3}{l}{$\begin{array}{l}\text { Regimen } \\
\text { Percentage of treated eyes with finding }(n)\end{array}$} \\
\cline { 2 - 4 } Sign/symptom & 1 Drop & $1+1$ Drop & $2+2$ Drop \\
\hline Ocular injection & $98(60)$ & $98(59)$ & $98(61)$ \\
Stinging & $31(19)$ & $48(29)$ & $44(27)$ \\
Burning & $20(12)$ & $28(17)$ & $29(18)$ \\
Lid oedema & $10(6)$ & $15(9)$ & $21(13)$ \\
Ptosis & $8(5)$ & $13(8)$ & $16(10)$ \\
Itching & $0(0)$ & $2(1)$ & $2(1)$ \\
Lid lag & $0(0)$ & $0(0)$ & $2(1)$ \\
Tearing & $0(0)$ & $0(0)$ & $2(1)$ \\
Vasodilatation & $0(0)$ & $0(0)$ & $2(1)$ \\
Conjunctivitis & $0(0)$ & $2(1)$ & $0(0)$ \\
$\quad$ (irritation) & $0(0)$ & $0(0)$ & $2(1)$ \\
Pain & & & \\
\hline
\end{tabular}


The subject's perception of the comfort of drops may influence the utility of the various regimens of dapiprazole. In this study, patient comfort appears to be enhanced, with generally fewer signs and symptoms occurring in association with both of the reduced dosage regimens. This reduction of signs and symptoms was most noticeable for burning, lid oedema, and ptosis.

Other factors influencing the use of an agent to reverse mydriasis include age, ocular pigmentation, and ocular health. Of those factors, eye colour analysis was a planned component of this study. Eye colour is known to affect mydriasis. Paggiarino ${ }^{10}$ reported that in a study of patients with blue or brown eyes, where mydriasis was induced by a combination of phenylephrine and tropicamide, $74 \%$ of the blue eyes returned to baseline pupil diameter within 5-7 hours, compared with $69 \%$ of the brown eyes. In this study, dapiprazole produced a slower effect on reversal of mydriasis in brown eyes, as have other $\alpha$ adrenergic blockers. ${ }^{2}$

In those instances where the clinician concludes that there is a practitioner benefit and/or a patient benefit to reversing mydriasis and enhancing recovery of accommodation, both the one drop regimen and the $1+1$ drop regimen appear to be safe, efficacious, and more convenient alternatives to the $2+2$ drop regimen. Indeed, in today's increasingly cost sensitive medical environment, the efficacy and safety of the one drop dapiprazole regimen seems to be a clinically advantageous treatment option for practitioners and their patients.

The authors acknowledge the assistance of Kathryn K Hart, MA in the statistical analysis of these data and Patricia Brown, $\mathrm{RN}$, MS for her editorial assistance in the preparation of this manuscript. Erika Jones was also instrumental in providing assistance in the revision processes.

Funding for this research was provided by Angelin Pharmaceuticals Inc, 70 Grand Avenue, River Edge, New Jersey 07661, USA.

1 Nyman N, Reich L. The effect of dapiprazole on accommodative amplitude in eyes dilated with 0.5 percent tropicamide. $\mathcal{f}$ Am Optom Assoc 1993; 64: 625-8.

2 Doughty MJ, Lyle WM. A review of the clinical pharmacokinetics of pilocarpine, moxisylyte (thymoxamine), and dapiprazole in the reversal of diagnostic pupillary dilation. Optom Vis Sci 1992; 69: 358-68.

3 MacKeen DL. Aqueous formulations and ointments. In Ophthalmol Clin 1980; 20: 79-92. Cited in Bartlett JD, Jaanus SD. Clinical ocular pharmacology. Boston: Butterworths, 1989.

4 Cline DH, Henry W, Griffin JR. Dictionary of visual science. 3rd ed. Radnor, PN, USA: Chilton Book Company, 1969; Vol 1, Ch 10: 11 .

5 Westlake WJ. Bioequivalence testing - a need to rethink. Biometrics 1981; 37: 591-3.

6 Connor CG, Campbell JB, Tirey WW. The clinical efficacy of Rev-Eyes in reversing the effects of pupillary dilation. f Am Optom Assoc 1993; 64: 634-6.

7 Johnson ME, Molinari JF, Carter J. Efficacy of dapiprazole with hydroxyamphetamine hydrobromide and tropicamide. F Am Optom Assoc 1993; 64: 629-33.

8 Allinson RW, Gerber DS, Bieber S, Hodes BL. Reversal of mydriasis by dapiprazole. Ann Ophthalmol 1990; 22: 131-8.

9 Paggiarino DA, Brancato LJ, Newton RE. The effects on pupil size and accommodation of sympathetic and purasympatholytic agents. Ann Ophthalmol 1993; 25: parasym

10 Nyman N, Keates EU. Effects of dapiprazole on the reversal of pharmacologically induced mydriasis. Optometry and Vision Science 1990; 67: 705-9. 